\title{
LOCAL MAXIMA OF A RANDOM ALGEBRAIC POLYNOMIAL
}

\author{
K. FARAHMAND and P. HANNIGAN
}

(Received 22 September 1999)

\begin{abstract}
We present a useful formula for the expected number of maxima of a normal process $\xi(t)$ that occur below a level $u$. In the derivation we assume chiefly that $\xi(t), \xi^{\prime}(t)$, and $\xi^{\prime \prime}(t)$ have, with probability one, continuous 1 dimensional distributions and expected values of zero. The formula referred to above is then used to find the expected number of maxima below the level $u$ for the random algebraic polynomial. This result highlights the very pronounced difference in the behaviour of the random algebraic polynomial on the interval $(-1,1)$ compared with the intervals $(-\infty,-1)$ and $(1, \infty)$. It is also shown that the number of maxima below the zero level is no longer $O(\log n)$ on the intervals $(-\infty,-1)$ and $(1, \infty)$.
\end{abstract}

2000 Mathematics Subject Classification. Primary 60H99, 26C99.

1. Introduction. A significant amount has been written concerning the mean number of crossings of a fixed level, by both stationary and nonstationary normal processes. Farahmand [5] has given a formula for the expected number of maxima, below a level $u$, for a normal process under certain assumptions. The initial interest in the stationary case can be traced back to Rice [13], and was subsequently investigated by Ito [7] and Ylvisarer [15]. These works concentrated on level crossings and have been reviewed in the comprehensive book by Cramér and Leadbetter [2]. In this book, Cramér and Leadbetter have also given a formula for the expected number of local maxima. In addition, their method enabled them to find the distribution function for the height of a local maximum. Leadbetter [9], in his treatment of the nonstationary case, gives a result for the mean number of level crossings by a normal process. In his work Leadbetter assumes that the random process has continuous sample functions, with probability one.

In what follows we consider $\xi(t)$ to be a real valued normal process. We assume $\xi(t)$, and its first and second derivatives $\xi^{\prime}(t)$ and $\xi^{\prime \prime}(t)$, posses, with probability one, continuous one dimensional distributions, such that the mean number of crossings of any level by $\xi(t)$, and the zero level by $\xi^{\prime}(t)$ are finite. In addition, we assume that the mean of $\xi(t), \xi^{\prime}(t)$, and $\xi^{\prime \prime}(t)$ are all zero. $\xi(t)$ has a local maximum at $t=t_{i}$, if $\xi^{\prime}(t)$ has a down crossing of the level zero at $t_{i}$. The local maxima which are of interest here, are those that occur when $\xi(t)$ is also below the level $u$. The total number of down crossings of the level zero by $\xi^{\prime}(t)$ in $(\alpha, \beta)$ is defined as $M(\alpha, \beta)$, and these occur at the points $\alpha<t_{1}<t_{2}<\cdots<t_{M(\alpha, \beta)}<\beta$. We define $M_{u}(\alpha, \beta)$ as the number of zero down crossings by $\xi^{\prime}(t)$, where $0 \leq i \leq M(\alpha, \beta)$ and $\xi\left(t_{i}\right) \leq u$. The corollary presented below is a corollary to Theorem 1.1 in [5]. The corollary is proved in Section 2. 
COROLLARY 1.1. For any random process $\xi(t)$ satisfying these conditions we denote the variances of $\xi(t), \xi^{\prime}(t)$, and $\xi^{\prime \prime}(t)$ by $A^{2} \equiv A^{2}(t), B^{2} \equiv B^{2}(t)$, and $C^{2} \equiv C^{2}(t)$, respectively, and let $D \equiv D(t), E \equiv E(t)$, and $F \equiv F(t)$ represent $\operatorname{cov}\left\{\xi(t), \xi^{\prime}(t)\right\}$, $\operatorname{cov}\left\{\xi(t), \xi^{\prime \prime}(t)\right\}$, and $\operatorname{cov}\left\{\xi^{\prime}(t), \xi^{\prime \prime}(t)\right\}$, respectively. Then the expected number of maxima below the level $u$, where $E\{\xi(t)\}=E\left\{\xi^{\prime}(t)\right\}=E\left\{\xi^{\prime \prime}(t)\right\}=0$, is given by

$$
\begin{aligned}
E M_{u}(\alpha, \beta)= & (2 \pi)^{-1} \int_{\alpha}^{\beta} d(2 e \sqrt{2 a})^{-1} \Phi(\sqrt{2 a} u) d t \\
& -(2 \pi)^{-1} \int_{\alpha}^{\beta} d(2 e \sqrt{2 a})^{-1}\left(\frac{b^{2}}{4 a c}\right)^{1 / 2} \Phi\left(u \sqrt{\frac{b^{2}}{2 c}}\right) \exp \left(-\frac{a e u^{2}}{c}\right) d t
\end{aligned}
$$

for $\left|\sum\right|=A^{2} B^{2} C^{2}-A^{2} F^{2}-B^{2} E^{2}-C^{2} D^{2}+2 D E F$,

$a=\frac{B^{2} C^{2}-F^{2}}{2\left|\sum\right|}, \quad b=\frac{D F-B^{2} E}{\left|\sum\right|}, \quad c=\frac{A^{2} B^{2}-D^{2}}{2\left|\sum\right|}, \quad d=\left|\sum\right|^{-1 / 2}, \quad e=c-\frac{b^{2}}{4 a}$.

The above corollary is useful for determining the total expected number of maxima of a normal process and also for finding the expected number of maxima of a random polynomial, in both cases these maxima occur below a level $u$. The result derived by Farahmand [5] for the random trigonometric polynomial is particularly suited to this treatment.

In this paper, we concentrate on the random algebraic polynomial

$$
T(x)=\sum_{j=0}^{n-1} a_{j}(\omega) x^{j} .
$$

We assume $a_{0}(\omega), a_{1}(\omega), \ldots, a_{n-1}(\omega)$ is a sequence of independent, normally distributed random variables defined on a probability space $(\Omega, A, \operatorname{Pr})$, each having mean zero and variance one. Let $M_{u}(\alpha, \beta)$ be the number of maxima of the polynomial $T(x)$ in the interval $(\alpha, \beta)$ below the level $u$, and let $E M_{u}(\alpha, \beta)$ be its mathematical expectation. We list a small number of relevant results for random polynomials below; a more complete reference can be found in the book by Bharucha-Reid and Sambandham [1]. Littlewood and Offord [10,11] initiated the study of random algebraic polynomials and Offord [12] continues this work in related fields. Kac [8] found the leading behaviour and an error term for the expected number of zero crossings of the random polynomial (1.3). Farahmand [4] found the expected number of $K$ level crossings in the interval $(-1,1)$ is asymptotic to $(1 / \pi) \log \left(n / K^{2}\right)$, and in the intervals $(-\infty,-1)$ and $(1, \infty)$ is asymptotic to $(1 / 2 \pi) \log n$, provided $K^{2} / n \rightarrow 0$ as $n \rightarrow \infty$. When $K^{2} / n$ tends to a nonzero constant Farahmand found the expected number of crossings to be asymptotic to $(1 / \pi) \log n$ for the interval $(-\infty, \infty)$. Wilkins [14] in recent times using a delicate method reduced the error term in Kac's result significantly. Das [3] considered the polynomial (1.3) with the same assumptions on the random coefficients. In this work, Das showed that the expected number of local maxima in the whole real line is asymptotic to $(\sqrt{3}+1) \log n / 2 \pi$. Farahmand and Hannigan [6] showed that if the random coefficients have nonzero mean, $\mu$, then the expected number of maxima is asymptotically half that of the Das result, this result remains valid when $\mu \rightarrow 0$ and 
$\mu \rightarrow \infty$. The expected number of maxima below a level $u$, for a random trigonometric polynomial with the same random coefficients as (1.3), was considered previously by Farahmand [5]. He showed that only about an eighth of the maxima appear below the level zero, or any level $u$ such that $u^{2} / n \rightarrow 0$ as $n \rightarrow \infty$.

In Theorem 1.2, we show that asymptotically all the maxima on the interval $(-1,1)$ occur below the level $u$, where $u \geq \sqrt{n}$. Also, we see that asymptotically around a fifth of the maxima occur below the level zero, or any level $u$ such that $u / \sqrt{\log n} \rightarrow 0$ as $n \rightarrow \infty$. On the intervals $(-\infty,-1)$ and $(1, \infty)$ the result is very different. In this case, asymptotically all the maxima on the intervals occur below the level $u \geq \exp (n / \log n)$. A seemingly strange result at first is that the number of maxima below the level zero, or any level $u$ such that $u / n^{k} \rightarrow 0$ as $n \rightarrow \infty$, is negligible compared with the number of maxima in all. We will show in the proof of Theorem 1.3 that $k$ can be any positive constant.

In Section 3, we will prove the following two theorems.

THEOREM 1.2. If the coefficients of $T(x)$ in (1.3) are independent, normally distributed random variables with mean zero and variance one, then for all sufficiently large $n$, and $u \equiv u_{n}$ defined below, the mathematical expectation of the number of maxima below the level $u$ satisfies

$$
\begin{aligned}
& E M_{u}(-1,0)=E M_{u}(0,1) \sim \frac{(\sqrt{3}-1) \log n}{8 \pi}, \quad \text { where } u=o(\sqrt{\log n}), \\
& E M_{u}(-1,0)=E M_{u}(0,1) \sim \frac{\sqrt{3} \log n}{4 \pi}, \quad \text { where } u \geq \sqrt{n} .
\end{aligned}
$$

THEOREM 1.3. For the same polynomial $T(x)$ defined above, and for all sufficiently large $n$, and $u \equiv u_{n}$ defined below, then

$$
\begin{aligned}
& E M_{u}(-\infty,-1)=E M_{u}(1, \infty)=o(\log n), \quad \text { where } u=o\left(n^{k}\right), \\
& E M_{u}(-\infty,-1)=E M_{u}(1, \infty) \sim \frac{\log n}{4 \pi}, \quad \text { where } u \geq \exp \left(\frac{n}{\log n}\right) .
\end{aligned}
$$

The second result in Theorem 1.2 is not surprising when we consider the results, outlined earlier, from Farahmand [4]. From Kac [8] and Das [3] we know that for the intervals $(-\infty,-1)$ and $(1, \infty)$ the expected number of both zero crossings and turning points are asymptotically equal. This suggests that asymptotically all the maxima are above the level zero; this partially explains the first result in Theorem 1.3.

2. Local maxima of a normal process. Farahmand proved in Theorem 1.1 of [5] that

$$
E M_{u}(\alpha, \beta)=\int_{\alpha}^{\beta} \int_{-\infty}^{u} \int_{-\infty}^{0}|z| p_{t}(x, 0, z) d z d x d t
$$

where $p_{t}(x, y, z)$ denotes the 3-dimensional normal density function for $\xi(t), \xi^{\prime}(t)$, and $\xi^{\prime \prime}(t)$. The 3-dimensional density function is based on the covariance matrix

$$
\sum=\left(\begin{array}{ccc}
A^{2} & D & E \\
D & B^{2} & F \\
E & F & C^{2}
\end{array}\right)
$$


in which

$$
\begin{gathered}
A^{2}=\operatorname{var}\{\xi(t)\}, \quad B^{2}=\operatorname{var}\left\{\xi^{\prime}(t)\right\}, \quad C^{2}=\operatorname{var}\left\{\xi^{\prime \prime}(t)\right\}, \\
D=\operatorname{cov}\left\{\xi(t), \xi^{\prime}(t)\right\}, \quad E=\operatorname{cov}\left\{\xi(t), \xi^{\prime \prime}(t)\right\}, \quad F=\operatorname{cov}\left\{\xi^{\prime}(t), \xi^{\prime \prime}(t)\right\},
\end{gathered}
$$

and the determinant of the covariance matrix, denoted $\left|\sum\right|$, is

$$
\left|\sum\right|=A^{2} B^{2} C^{2}-A^{2} F^{2}-B^{2} E^{2}-C^{2} D^{2}+2 D E F .
$$

We define $p_{t}(x, 0, z)$, not in terms of the variables above, but with the more convenient notation

$$
a=\frac{B^{2} C^{2}-F^{2}}{2\left|\sum\right|}, \quad b=\frac{D F-B^{2} E}{\left|\sum\right|}, \quad c=\frac{A^{2} B^{2}-D^{2}}{2\left|\sum\right|}, \quad d=\left|\sum\right|^{-1 / 2} .
$$

That is,

$$
p_{t}(x, 0, z)=(2 \pi)^{-3 / 2} d \exp \left\{-a x^{2}-b x z-c z^{2}\right\} .
$$

Combining (2.1) and (2.6) we find that

$$
E M_{u}(\alpha, \beta)=\int_{\alpha}^{\beta} \int_{-\infty}^{u} \int_{-\infty}^{0}(2 \pi)^{-3 / 2} d \exp \left\{-a x^{2}-b x z-c z^{2}\right\} d z d x d t .
$$

By splitting the exponential element in (2.7) into two parts, one of which isolates $x$, we have

$$
\begin{aligned}
E M_{u}(\alpha, \beta)= & (2 \pi)^{-3 / 2} \int_{\alpha}^{\beta} d \int_{-\infty}^{0}-z \exp \left\{-\left(c-\frac{b^{2}}{4 a}\right) z^{2}\right\} \\
& \times \int_{-\infty}^{u} \exp \left\{-\frac{(\sqrt{2 a} x+b z / \sqrt{2 a})^{2}}{2}\right\} d x d z d t .
\end{aligned}
$$

It is possible now to rewrite the portion of (2.8) referring to $x$, in terms of $v$; that is, $v=\sqrt{2 a} x+b z / \sqrt{2 a}$. It also simplifies our analysis if we let

$$
e=c-\frac{b^{2}}{4 a}
$$

Therefore,

$$
\begin{aligned}
E M_{u}(\alpha, \beta)= & (2 \pi)^{-3 / 2} \int_{\alpha}^{\beta} \frac{d}{\sqrt{2 a}} \int_{-\infty}^{0}-z \exp \left\{-e z^{2}\right\} \\
& \times \int_{-\infty}^{\sqrt{2 a} u+b z / \sqrt{2 a}} \exp \left\{-\frac{v^{2}}{2}\right\} d v d z d t
\end{aligned}
$$

At this point we interchange the order of the integration in (2.10) with respect to the variables $v$ and $z$, thus

$$
\begin{aligned}
E M_{u}(\alpha, \beta)= & (2 \pi)^{-3 / 2} \int_{\alpha}^{\beta} \frac{d}{\sqrt{2 a}} \int_{-\infty}^{\sqrt{2 a} u} \exp \left\{-\frac{v^{2}}{2}\right\} \\
& \times \int_{\sqrt{2 a}(v-\sqrt{2 a} u) / b}^{0}-z \exp \left\{-e z^{2}\right\} d z d v d t .
\end{aligned}
$$


We can integrate with respect to $z$ in (2.11) to find that

$$
\begin{aligned}
E M_{u}(\alpha, \beta)= & (2 \pi)^{-3 / 2} \int_{\alpha}^{\beta} d(2 e \sqrt{2 a})^{-1}\left(\int_{-\infty}^{\sqrt{2 a} u} \exp \left\{-\frac{v^{2}}{2}\right\} d v\right. \\
& \left.-\int_{-\infty}^{\sqrt{2 a} u} \exp \left\{-\frac{v^{2}}{2}-\frac{2 a e(v-\sqrt{2 a} u)^{2}}{b^{2}}\right\} d v\right) d t .
\end{aligned}
$$

After a little algebraic manipulation we can separate the exponential in the second integral of (2.12) into two parts, that is

$$
\begin{aligned}
& \exp \left\{-\frac{v^{2}}{2}-\frac{2 a e(v-\sqrt{2 a} u)^{2}}{b^{2}}\right\} \\
& \quad=\exp \left\{-\frac{\left[\left(\left(b^{2}+4 a e\right) / b^{2}\right)^{1 / 2} v-\left(32 a^{3} e^{2} /\left(b^{4}+4 a b^{2} e\right)\right)^{1 / 2} u\right]^{2}}{2}\right\} \exp \left\{-\frac{a e u^{2}}{c}\right\} .
\end{aligned}
$$

Using (2.13) and integration by substitution, together with our knowledge of the probability distribution function $\Phi(x)$ we can rewrite (2.12) as

$$
\begin{aligned}
E M_{u}(\alpha, \beta)= & (2 \pi)^{-1} \int_{\alpha}^{\beta} d(2 e \sqrt{2 a})^{-1} \Phi(\sqrt{2 a} u) d t \\
& -(2 \pi)^{-1} \int_{\alpha}^{\beta} d(2 e \sqrt{2 a})^{-1}\left(\frac{b^{2}}{4 a c}\right)^{1 / 2} \Phi\left(u \sqrt{\frac{b^{2}}{2 c}}\right) \exp \left(-\frac{a e u^{2}}{c}\right) d t .
\end{aligned}
$$

This completes the proof of the corollary.

3. Proof of theorems for the random algebraic polynomial. In order to find $E M_{u}$ $(\alpha, \beta)$ for the random algebraic polynomial (1.3), we principally use the result in Corollary 1.1. We also use a result due to Das [3].

Applying Corollary 1.1 to the polynomial (1.3), we find

$$
\begin{aligned}
E M_{u}(\alpha, \beta)= & (2 \pi)^{-1} \int_{\alpha}^{\beta} d(2 e \sqrt{2 a})^{-1} \Phi(\sqrt{2 a} u) d x \\
& -(2 \pi)^{-1} \int_{\alpha}^{\beta} d(2 e \sqrt{2 a})^{-1}\left(\frac{b^{2}}{4 a c}\right)^{1 / 2} \Phi\left(u \sqrt{\frac{b^{2}}{2 c}}\right) \exp \left(-\frac{a e u^{2}}{c}\right) d x \\
= & \int_{\alpha}^{\beta} I_{1}(x) d x-\int_{\alpha}^{\beta} I_{2}(x) d x,
\end{aligned}
$$

where

$$
\begin{gathered}
A^{2}=\operatorname{var}\{T(x)\}, \quad B^{2}=\operatorname{var}\left\{T^{\prime}(x)\right\}, \quad C^{2}=\operatorname{var}\left\{T^{\prime \prime}(x)\right\}, \\
D=\operatorname{cov}\left\{T(x), T^{\prime}(x)\right\}, \quad E=\operatorname{cov}\left\{T(x), T^{\prime \prime}(x)\right\}, \quad F=\operatorname{cov}\left\{T^{\prime}(x), T^{\prime \prime}(x)\right\},
\end{gathered}
$$

and

$$
\begin{gathered}
\left|\sum\right|=A^{2} B^{2} C^{2}-A^{2} F^{2}-B^{2} E^{2}-C^{2} D^{2}+2 D E F, \\
a=\frac{B^{2} C^{2}-F^{2}}{2\left|\sum\right|}, \quad b=\frac{D F-B^{2} E}{\left|\sum\right|}, \quad c=\frac{A^{2} B^{2}-D^{2}}{2\left|\sum\right|}, \quad d=\left|\sum\right|^{-1 / 2} .
\end{gathered}
$$


Since the coefficients of $T(x)$ in (1.3) are independent, normally distributed random variables with mean zero and variance one, it is a trivial task to show that

$$
\begin{array}{cc}
A^{2}=\sum_{j=0}^{n-1} x^{2 j}, & B^{2}=\sum_{j=0}^{n-1} j^{2} x^{2 j-2}, \quad C^{2}=\sum_{j=0}^{n-1} j^{2}(j-1)^{2} x^{2 j-4}, \\
D=\sum_{j=0}^{n-1} j x^{2 j-1}, & E=\sum_{j=0}^{n-1} j(j-1) x^{2 j-2}, \quad F=\sum_{j=0}^{n-1} j^{2}(j-1) x^{2 j-3} .
\end{array}
$$

By repeated differentiation of the geometric sum

$$
\sum_{j=0}^{n-1} x^{2 j}=\frac{1-x^{2 n}}{1-x^{2}}
$$

we obtain

$$
\begin{aligned}
A^{2}= & \frac{x^{2 n}-1}{x^{2}-1}, \\
B^{2}= & \frac{n^{2} x^{2 n-2}}{x^{2}-1}-\frac{2 n x^{2 n}}{\left(x^{2}-1\right)^{2}}+\frac{\left(1+x^{2}\right)\left(x^{2 n}-1\right)}{\left(x^{2}-1\right)^{3}}, \\
C^{2}= & \frac{n^{4} x^{2 n-4}}{x^{2}-1}-\frac{6 n^{3} x^{2 n-2}-2 n^{3} x^{2 n-4}}{\left(x^{2}-1\right)^{2}} \\
& +\frac{13 n^{2} x^{2 n}-2 n^{2} x^{2 n-2}+n^{2} x^{2 n-4}}{\left(x^{2}-1\right)^{3}}+\frac{12 x^{2 n}\left(x^{2}+1\right)}{\left(x^{2}-1\right)^{4}}+\frac{\left(x^{2 n}-1\right)\left(4 x^{4}+16 x^{2}+4\right)}{\left(x^{2}-1\right)^{5}}, \\
D= & \frac{n x^{2 n-1}}{x^{2}-1}-\frac{x\left(x^{2 n}-1\right)}{\left(x^{2}-1\right)^{2}}, \\
E= & \frac{n^{2} x^{2 n-2}}{x^{2}-1}-\frac{3 n x^{2 n}-n x^{2 n-2}}{\left(x^{2}-1\right)^{2}}+\frac{2 x^{2}\left(x^{2 n}-1\right)}{\left(x^{2}-1\right)^{3}}, \\
F= & \frac{n^{3} x^{2 n-3}}{x^{2}-1}-\frac{n^{2} x^{2 n-3}\left(4 x^{2}-1\right)}{\left(x^{2}-1\right)^{2}}+\frac{n x^{2 n-1}\left(5 x^{2}+1\right)}{\left(x^{2}-1\right)^{3}}-\frac{2 x^{2 n+3}+4 x^{2 n+1}-2 x^{3}-4 x}{\left(x^{2}-1\right)^{4}} .
\end{aligned}
$$

Looking at the properties of the random coefficients in (1.3) it is clear that $E M_{u}(-b,-a)$ $=E M_{u}(a, b)$. Obviously, we need only find the leading behaviour for $E M_{u}(0,1)$ and $E M_{u}(1, \infty)$ to prove the two theorems.

To this end we look initially at the interval $0<x<1-\epsilon$, where $\epsilon_{n} \equiv \epsilon=n^{-\alpha}$, and $\alpha$ is any positive value, satisfying

$$
\alpha=\frac{1-\log \log n^{k}}{\log n}
$$

The positive constant $k$ is critical to these theorems and we will return to define a set of qualifying $k$ values later. From (3.6), and since for all $n$ sufficiently large, 
$x^{2 n}<\left(1-n^{-\alpha}\right)^{2 n}<\exp \left(-2 n^{1-\alpha}\right)$ we have

$$
\begin{aligned}
& A^{2}=\left(1-x^{2}\right)^{-1}\left(1+O\left\{\exp \left(-2 n^{1-\alpha}\right)\right\}\right), \\
& B^{2}=\left(1+x^{2}\right)\left(1-x^{2}\right)^{-3}\left(1+O\left\{n^{2} \exp \left(-2 n^{1-\alpha}\right)\right\}\right), \\
& C^{2}=\left(4+16 x^{2}+4 x^{4}\right)\left(1-x^{2}\right)^{-5}\left(1+O\left\{n^{4} \exp \left(-2 n^{1-\alpha}\right)\right\}\right), \\
& D=x\left(1-x^{2}\right)^{-2}\left(1+O\left\{n \exp \left(-2 n^{1-\alpha}\right)\right\}\right), \\
& E=2 x^{2}\left(1-x^{2}\right)^{-3}\left(1+O\left\{n^{2} \exp \left(-2 n^{1-\alpha}\right)\right\}\right), \\
& F=\left(4 x+2 x^{3}\right)\left(1-x^{2}\right)^{-4}\left(1+O\left\{n^{3} \exp \left(-2 n^{1-\alpha}\right)\right\}\right) .
\end{aligned}
$$

Using (3.8) and the definitions we have for $a, b, c, d, e$, and $\left|\sum\right|$ it is a reasonably simple task to show that

$$
\begin{aligned}
& a=\frac{\left(1-x^{6}\right)\left(1+O\left\{n^{4} \exp \left(-2 n^{1-\alpha}\right)\right\}\right)}{2}, \\
& b=\frac{x^{2}\left(1-x^{2}\right)^{3}\left(1+O\left\{n^{4} \exp \left(-2 n^{1-\alpha}\right)\right\}\right)}{2}, \\
& c=\frac{\left(1-x^{2}\right)^{5}\left(1+O\left\{n^{4} \exp \left(-2 n^{1-\alpha}\right)\right\}\right)}{8}, \\
& d=\frac{\left(1-x^{2}\right)^{9 / 2}\left(1+O\left\{n^{4} \exp \left(-2 n^{1-\alpha}\right)\right\}\right)}{2}, \\
& e=\frac{\left(1-x^{2}\right)^{5}\left(1-x^{4}\right)\left(1-x^{6}\right)^{-1}\left(1+O\left\{n^{4} \exp \left(-2 n^{1-\alpha}\right)\right\}\right)}{8} .
\end{aligned}
$$

Employing our definition of $\alpha$ in (3.7) we can show

$$
\exp \left(-2 n^{1-\alpha}\right)=\exp \left(-2 \log n^{k}\right)=n^{-2 k},
$$

for all sufficiently large $n$, all terms inside $O\{\}$ in (3.8) and (3.9) will tend to zero if $k$ is any number greater than 2. Using (3.1) and (3.9) we have that

$$
\int_{0}^{1-\epsilon} I_{1}(x) d x \sim \int_{0}^{1-n^{-\alpha}}\left(1+x^{2}+x^{4}\right)^{1 / 2}\left(1-x^{4}\right)^{-1} \Phi\left\{\left(1-x^{6}\right)^{1 / 2} u\right\} d x .
$$

Obviously,

$$
\begin{aligned}
& \pi^{-1} \int_{1-(\log n)^{-1}}^{1-n^{-\alpha}}\left(1+x^{2}+x^{4}\right)^{1 / 2}\left(1-x^{4}\right)^{-1} \Phi\left\{\left(1-x^{6}\right)^{1 / 2} u\right\} d x \\
& \quad \leq \int_{0}^{1-\epsilon} I_{1}(x) d x \\
& \quad \leq \pi^{-1} \int_{0}^{1-(\log n)^{-1}}\left(1+x^{2}+x^{4}\right)^{1 / 2}\left(1-x^{4}\right)^{-1} d x \\
& \quad+\pi^{-1} \int_{1-(\log n)^{-1}}^{1-n^{-\alpha}}\left(1+x^{2}+x^{4}\right)^{1 / 2}\left(1-x^{4}\right)^{-1} \Phi\left\{\left(1-x^{6}\right)^{1 / 2} u\right\} d x .
\end{aligned}
$$

Concentrating on the integral common to both sides of the composite inequality (3.12) and using the fact that $\Phi\left\{\left(1-x^{6}\right)^{1 / 2} u\right\}$ is a decreasing function on the interval 
$\left(1-(\log n)^{-1}, 1-n^{-\alpha}\right)$, it can be shown that

$$
\begin{aligned}
\pi^{-1} & \left.\Phi\left(\frac{6}{n^{\alpha}}\right)^{1 / 2} u\right\} \int_{1-(\log n)^{-1}}^{1-n^{-\alpha}}\left(1+x^{2}+x^{4}\right)^{1 / 2}\left(1-x^{4}\right)^{-1} d x \\
& \leq \pi^{-1} \int_{1-(\log n)^{-1}}^{1-n^{-\alpha}}\left(1+x^{2}+x^{4}\right)^{1 / 2}\left(1-x^{4}\right)^{-1} \Phi\left\{\left(1-x^{6}\right)^{1 / 2} u\right\} d x \\
& \leq \pi^{-1} \Phi\left\{\left(\frac{6}{\log n}\right)^{1 / 2} u\right\} \int_{1-(\log n)^{-1}}^{1-n^{-\alpha}}\left(1+x^{2}+x^{4}\right)^{1 / 2}\left(1-x^{4}\right)^{-1} d x .
\end{aligned}
$$

For some $\delta \equiv \delta_{n}$, such that $0<\delta_{n}<1$, and $\lim _{n \rightarrow \infty} \delta_{n}=0$, we can see that

$$
\begin{aligned}
\left(\frac{\sqrt{3}}{\pi}\right) \int_{0}^{1-\delta} x^{2}\left(1-x^{4}\right)^{-1} d x & \leq \pi^{-1} \int_{0}^{1-\delta}\left(1+x^{2}+x^{4}\right)^{1 / 2}\left(1-x^{4}\right)^{-1} d x \\
& \leq\left(\frac{\sqrt{3}}{\pi}\right) \int_{0}^{1-\delta}\left(1-x^{4}\right)^{-1} d x .
\end{aligned}
$$

Using partial fraction expansions in (3.14) we can quickly show that

$$
\pi^{-1} \int_{0}^{1-\delta}\left(1+x^{2}+x^{4}\right)^{1 / 2}\left(1-x^{4}\right)^{-1} d x \sim \frac{\sqrt{3} \log (1 / \delta)}{4 \pi} .
$$

Hence, using (3.11), (3.12), (3.13), (3.14), and (3.15) it becomes clear that

$$
\begin{aligned}
& \int_{0}^{1-\epsilon} I_{1}(x) d x \sim \frac{\sqrt{3} \log n}{8 \pi}, \quad \text { where } u=o(\sqrt{\log n}), \\
& \int_{0}^{1-\epsilon} I_{1}(x) d x \sim \frac{\sqrt{3} \log n}{4 \pi}, \quad \text { where } u \geq \sqrt{n^{\alpha}} f,
\end{aligned}
$$

if $f$ is any function of $n$ such that it tends to infinity as $n$ tends to infinity. The condition on $u$ in (3.17) can be greatly simplified, leading to a more meaningful result, by increasing the level $u$ marginally, to yield

$$
\int_{0}^{1-\epsilon} I_{1}(x) d x \sim \frac{\sqrt{3} \log n}{4 \pi}, \quad \text { where } u \geq \sqrt{n} .
$$

At this juncture we turn our attention to the integral of $I_{2}(x)$ and we employ many of the techniques previously used. Using (3.1) and (3.9),

$$
\int_{0}^{1-\epsilon} I_{2}(x) d x \sim \pi^{-1} \int_{0}^{1-n^{-\alpha}} x^{2}\left(1-x^{4}\right)^{-1} \Phi\left\{x^{2}\left(1-x^{2}\right)^{1 / 2} u\right\} \exp \left\{-\frac{\left(1-x^{4}\right) u^{2}}{2}\right\} d x .
$$

Obviously,

$$
\begin{aligned}
& \pi^{-1} \int_{1-(\log n)^{-1}}^{1-n^{-\alpha}} x^{2}\left(1-x^{4}\right)^{-1} \Phi\left\{x^{2}\left(1-x^{2}\right)^{1 / 2} u\right\} \exp \left\{-\frac{\left(1-x^{4}\right) u^{2}}{2}\right\} d x \\
& \quad \leq \int_{0}^{1-\epsilon} I_{2}(x) d x \\
& \quad \leq \pi^{-1} \int_{0}^{1-(\log n)^{-1}} x^{2}\left(1-x^{4}\right)^{-1} \exp \left\{-\frac{\left(1-x^{4}\right) u^{2}}{2}\right\} d x \\
& \quad+\pi^{-1} \int_{1-(\log n)^{-1}}^{1-n^{-\alpha}} x^{2}\left(1-x^{4}\right)^{-1} \Phi\left\{x^{2}\left(1-x^{2}\right)^{1 / 2} u\right\} \exp \left\{-\frac{\left(1-x^{4}\right) u^{2}}{2}\right\} d x
\end{aligned}
$$


As in the integral of $I_{1}(x)$, the integral common to both sides of the double inequality (3.20), is critical to our proof. Since $x^{2}\left(1-x^{2}\right)^{1 / 2}$ has a maximum at $(1 / 3)^{1 / 3}$, it is clear that $\Phi\left\{x^{2}\left(1-x^{2}\right)^{1 / 2} u\right\}$ is decreasing on the interval $\left(1-(\log n)^{-1}, 1-n^{-\alpha}\right)$. It is also evident that $\exp \left\{-\left(1-x^{4}\right) u^{2} / 2\right\}$ is an increasing function for $x \in(0,1)$. Combining our knowledge of $\Phi\left\{x^{2}\left(1-x^{2}\right)^{1 / 2} u\right\}$ and $\exp \left\{-\left(1-x^{4}\right) u^{2} / 2\right\}$ it is clear that

$$
\begin{aligned}
& \pi^{-1} \Phi\left\{\left(\frac{2}{n^{\alpha}}\right)^{1 / 2} u\right\} \exp \left\{-\frac{2 u^{2}}{\log n}\right\} \int_{1-(\log n)^{-1}}^{1-n^{-\alpha}} x^{2}\left(1-x^{4}\right)^{-1} d x \\
& \quad \leq \pi^{-1} \int_{1-(\log n)^{-1}}^{1-n^{-\alpha}} x^{2}\left(1-x^{4}\right)^{-1} \Phi\left\{x^{2}\left(1-x^{2}\right)^{1 / 2} u\right\} \exp \left\{-\frac{\left(1-x^{4}\right) u^{2}}{2}\right\} d x \\
& \quad \leq \pi^{-1} \Phi\left\{\left(\frac{2}{\log n}\right)^{1 / 2} u\right\} \exp \left\{-\frac{2 u^{2}}{n^{\alpha}}\right\} \int_{1-(\log n)^{-1}}^{1-n^{-\alpha}} x^{2}\left(1-x^{4}\right)^{-1} d x
\end{aligned}
$$

Simply by integrating the terms in (3.21) we find

$$
\begin{aligned}
& (4 \pi)^{-1} \Phi\left\{\left(\frac{2}{n^{\alpha}}\right)^{1 / 2} u\right\} \exp \left\{-\frac{2 u^{2}}{\log n}+\log \log n\right\} \\
& \quad \leq \pi^{-1} \int_{1-(\log n)^{-1}}^{1-n^{-\alpha}} x^{2}\left(1-x^{4}\right)^{-1} \Phi\left\{x^{2}\left(1-x^{2}\right)^{1 / 2} u\right\} \exp \left\{-\frac{\left(1-x^{4}\right) u^{2}}{2}\right\} d x \\
& \quad \leq(4 \pi)^{-1} \Phi\left\{\left(\frac{2}{\log n}\right)^{1 / 2} u\right\} \exp \left\{-\frac{2 u^{2}}{\log n}+\log \log n\right\} .
\end{aligned}
$$

Hence, using (3.20) and (3.22) it is clear that

$$
\begin{aligned}
& \int_{0}^{1-\epsilon} I_{2}(x) d x \sim \frac{\log n}{8 \pi}, \quad \text { where } u=o(\sqrt{\log n}) \\
& \int_{0}^{1-\epsilon} I_{2}(x) d x \rightarrow 0, \quad \text { where } u \geq \sqrt{n}
\end{aligned}
$$

From (3.16), (3.18), (3.23), and (3.24) we have proved Theorem 1.2 on the intervals $(-1+\epsilon, 0)$ and $(0,1-\epsilon)$. We will use a result due to Das [3] to complete the proof of the theorem. From (3.18) and (3.24) it is clear that

$$
E M_{u}(0,1-\epsilon) \sim \frac{\sqrt{3} \log n}{4 \pi}, \quad \text { where } u \geq \sqrt{n} .
$$

This is the same asymptotic value that Das [3] obtained for the number of maxima on the interval $(0,1)$. Therefore, $E M_{u}(1-\epsilon, 1)=o(\log n)$, irrespective of the level $u$ and the theorem is proved.

In Theorem 1.3, we want to evaluate $E M_{u}(1, \infty)$ asymptotically. It becomes apparent later that we need only find $E M_{u}(1+\varepsilon, \infty)$ (where $\varepsilon$ is defined below), and it proves convenient to make the substitution $y=1 / x$. If we let $1+\varepsilon=(1-\epsilon)^{-1}$, where $\epsilon=n^{-\alpha}$ as before. It will be necessary to define a set of qualifying $k$ values for (3.7). We make 
the substitution in (3.6) to find

$$
\begin{aligned}
A^{2}=y^{-2 n+2}\left(1-y^{2}\right)^{-1}\{1 & \left.+O\left\{\exp \left(-2 n^{1-\alpha}\right)\right\}\right\}, \\
B^{2}=n^{2} y^{-2 n+4}\left(1-y^{2}\right)^{-1}\left\{1-2 n^{-1}\left(1-y^{2}\right)^{-1}+\left(1+y^{2}\right) n^{-2}\left(1-y^{2}\right)^{-2}\right. & \left.+O\left\{n^{-2+2 \alpha} \exp \left(-2 n^{1-\alpha}\right)\right\}\right\}, \\
C^{2}=n^{4} y^{-2 n+6}\left(1-y^{2}\right)^{-1}\{1 & +\left(-6+2 y^{2}\right) n^{-1}\left(1-y^{2}\right)^{-1} \\
& \left(13-2 y^{2}+y^{4}\right) n^{-2}\left(1-y^{2}\right)^{-2}-12\left(1+y^{2}\right) n^{-3}\left(1-y^{2}\right)^{-3} \\
& \left.+\left(4+16 y^{2}+4 y^{4}\right) n^{-4}\left(1-y^{2}\right)^{-4}+O\left\{n^{-4+4 \alpha} \exp \left(-2 n^{1-\alpha}\right)\right\}\right\}, \\
D=n y^{-2 n+3}\left(1-y^{2}\right)^{-1}\{1 & \left.-n^{-1}\left(1-y^{2}\right)^{-1}+O\left\{n^{-1+\alpha} \exp \left(-2 n^{1-\alpha}\right)\right\}\right\}, \\
E=n^{2} y^{-2 n+4}\left(1-y^{2}\right)^{-1}\{1 & +\left(-3+y^{2}\right) n^{-1}\left(1-y^{2}\right)^{-1}+2 n^{-2}\left(1-y^{2}\right)^{-2} \\
+ & \left.O\left\{n^{-2+2 \alpha} \exp \left(-2 n^{1-\alpha}\right)\right\}\right\}, \\
F=n^{3} y^{-2 n+5}\left(1-y^{2}\right)^{-1}\{1 & -\left(4-y^{2}\right) n^{-1}\left(1-y^{2}\right)^{-1}+\left(5+y^{2}\right) n^{-2}\left(1-y^{2}\right)^{-2} \\
- & \left.\left(2+4 y^{2}\right) n^{-3}\left(1-y^{2}\right)^{-3}+O\left\{n^{-3+3 \alpha} \exp \left(-2 n^{1-\alpha}\right)\right\}\right\} .
\end{aligned}
$$

Using (3.26) and our definition for $a, b, c, d, e$, and $\left|\sum\right|$ it can be shown that

$$
\begin{aligned}
& a=n^{4}\left(1-y^{2}\right)^{5} y^{2 n}\left(8 y^{6}\right)^{-1}\left\{1+\left(-6-2 y^{2}\right) n^{-1}\left(1-y^{2}\right)^{-1}+\left(13+10 y^{2}+y^{4}\right) n^{-2}\left(1-y^{2}\right)^{-2}\right. \\
& +\left(-12-12 y^{2}\right) n^{-3}\left(1-y^{2}\right)^{-3}+\left(4+4 y^{2}+4 y^{4}\right) n^{-4}\left(1-y^{2}\right)^{-4} \\
& \left.+O\left\{\exp \left(-2 n^{1-\alpha}\right)\right\}\right\} \text {, } \\
& b=n^{2} y^{2 n-2}\left(1-y^{2}\right)^{5}\left(4 y^{6}\right)^{-1}\left\{1+\left(-3-y^{2}\right) n^{-1}\left(1-y^{2}\right)^{-1}+2 n^{-2}\left(1-y^{2}\right)^{-2}\right. \\
& \left.+O\left\{\exp \left(-2 n^{1-\alpha}\right)\right\}\right\}, \\
& c=y^{2 n-4}\left(1-y^{2}\right)^{5}\left(8 y^{6}\right)^{-1}\left\{1+O\left\{\exp \left(-2 n^{1-\alpha}\right)\right\}\right\}, \\
& d=y^{3 n-6}\left(1-y^{2}\right)^{9 / 2}\left(2 y^{3}\right)^{-1}\left\{1+O\left\{\exp \left(-2 n^{1-\alpha}\right)\right\}\right\}, \\
& e=y^{2 n-4}\left(1-y^{2}\right)^{3}\left(2 n^{2} y^{2}\right)^{-1}\left\{1+O\left\{\exp \left(-2 n^{1-\alpha}\right)\right\}\right\} .
\end{aligned}
$$

Since we are using the definition of $\alpha$ given in (3.7), it is clear that $k$ can be any positive constant in this case.

Employing (3.1) and (3.27) it is clear that

$$
\int_{1+\varepsilon}^{\infty} I_{1}(x) d x \sim(2 \pi)^{-1} \int_{0}^{1-\epsilon} y^{-2}\left(1-y^{2}\right)^{-1} \Phi\left\{\frac{n^{2}\left(1-y^{2}\right)^{5 / 2} y^{n-3} u}{2}\right\} d y .
$$

It can easily be shown that $\Phi\{\}$ in (3.28) is an increasing function on the interval $(0,1-\epsilon)$. In much the same way as we proved Theorem 1.2 , we see that 


$$
\begin{aligned}
(2 \pi)^{-1} & \int_{1-(\log n)^{-1}}^{1-n^{-\alpha}} y^{-2}\left(1-y^{2}\right)^{-1} \Phi\left\{\frac{n^{2}\left(1-y^{2}\right)^{5 / 2} y^{n-3} u}{2}\right\} d y \\
\leq & \int_{1+\varepsilon}^{\infty} I_{1}(x) d x \\
\leq & (2 \pi)^{-1} \int_{0}^{1-(\log n)^{-1}} y^{-2}\left(1-y^{2}\right)^{-1} d y \\
& +(2 \pi)^{-1} \int_{1-(\log n)^{-1}}^{1-n^{-\alpha}} y^{-2}\left(1-y^{2}\right)^{-1} \Phi\left\{\frac{n^{2}\left(1-y^{2}\right)^{5 / 2} y^{n-3} u}{2}\right\} d y .
\end{aligned}
$$

As in Theorem 1.2, we concentrate on the integral in the interval $\left(1-(\log n)^{-1}, 1-n^{-\alpha}\right)$ and use the fact that $\Phi\{\}$ is an increasing function

$$
\begin{aligned}
(2 \pi)^{-1} & \left\{\frac{2 \sqrt{2} n^{2} \exp (-n / \log n) u}{(\log n)^{5 / 2}}\right\} \int_{1-(\log n)^{-1}}^{1-n^{-\alpha}} y^{-2}\left(1-y^{2}\right)^{-1} d y \\
& \leq(2 \pi)^{-1} \int_{1-(\log n)^{-1}}^{1-n^{-\alpha}} y^{-2}\left(1-y^{2}\right)^{-1} \Phi\left\{\frac{n^{2}\left(1-y^{2}\right)^{5 / 2} y^{n-3} u}{2}\right\} d y \\
& \leq(2 \pi)^{-1} \Phi\left\{2 \sqrt{2} n^{2} \exp \frac{\left(-n^{1-\alpha}\right) u}{n^{5 \alpha / 2}}\right\} \int_{1-(\log n)^{-1}}^{1-n^{-\alpha}} y^{-2}\left(1-y^{2}\right)^{-1} d y .
\end{aligned}
$$

Hence, using (3.29), (3.30), and a little simple integration it is clear that

$$
\begin{aligned}
& \int_{1+\varepsilon}^{\infty} I_{1}(x) d x \sim \frac{\log n}{8 \pi}, \quad \text { where } u=o\left(n^{k}\right), \\
& \int_{1+\varepsilon}^{\infty} I_{1}(x) d x \sim \frac{\log n}{4 \pi}, \quad \text { where } u \geq \frac{\exp (n / \log n)(\log n)^{5 / 2} f}{n^{2}},
\end{aligned}
$$

if $f$ is any function which tends to infinity as $n$ tends to infinity.

The only significant part to prove now is the integral of $I_{2}(x)$ on the interval $\left((1+\epsilon)^{-1}, \infty\right)$. From (3.1) and (3.27) we can show

$$
\begin{aligned}
\int_{1+\varepsilon}^{\infty} I_{2}(x) d x \sim(2 \pi)^{-1} \int_{0}^{1-\epsilon} & y^{-2}\left(1-y^{2}\right)^{-1} \exp \left\{-\frac{n^{2}\left(1-y^{2}\right)^{3} y^{2 n-2} u^{2}}{2}\right\} \\
& \times \Phi\left\{\frac{n^{2}\left(1-y^{2}\right)^{5 / 2} y^{n-3} u}{2}\right\} d y .
\end{aligned}
$$

By calculus methods we can prove that $\exp \{\}$ in (3.33) is a decreasing function on the interval $(0,1-\epsilon)$. The $\Phi\{\}$ element in (3.33) is identical to the $\Phi\{\}$ in (3.29). In much the same way as before

$$
\begin{gathered}
(2 \pi)^{-1} \int_{1-(\log n)^{-1}}^{1-n^{-\alpha}} y^{-2}\left(1-y^{2}\right)^{-1} \exp \left\{-\frac{n^{2}\left(1-y^{2}\right)^{3} y^{2 n-2} u^{2}}{2}\right\} \Phi\left\{\frac{n^{2}\left(1-y^{2}\right)^{5 / 2} y^{n-3} u}{2}\right\} d y \\
\leq \int_{1+\varepsilon}^{\infty} I_{2}(x) d x \\
\leq(2 \pi)^{-1} \int_{0}^{1-(\log n)^{-1}} y^{-2}\left(1-y^{2}\right)^{-1} \exp \left\{-\frac{n^{2}\left(1-y^{2}\right)^{3} y^{2 n-2} u^{2}}{2}\right\} d y \\
+(2 \pi)^{-1} \int_{1-(\log n)^{-1}}^{1-n^{-\alpha}} y^{-2}\left(1-y^{2}\right)^{-1} \exp \left\{-\frac{n^{2}\left(1-y^{2}\right)^{3} y^{2 n-2} u^{2}}{2}\right\} \\
\times \Phi\left\{\frac{n^{2}\left(1-y^{2}\right)^{5 / 2} y^{n-3} u}{2}\right\} d y .
\end{gathered}
$$


Also,

$$
\begin{aligned}
(2 \pi)^{-1} & \exp \left\{-\frac{4 n^{2} \exp \left(-2 n^{1-\alpha}\right) u^{2}}{n^{3 \alpha}}\right\} \Phi\left\{\frac{2 \sqrt{2} n^{2} \exp (-n / \log n) u}{(\log n)^{5 / 2}}\right\} \\
& \times \int_{1-(\log n)^{-1}}^{1-n^{-\alpha}} y^{-2}\left(1-y^{2}\right)^{-1} d y \\
\leq & (2 \pi)^{-1} \int_{1-(\log n)^{-1}}^{1-n^{-\alpha}} y^{-2}\left(1-y^{2}\right)^{-1} \exp \left\{-\frac{n^{2}\left(1-y^{2}\right)^{3} y^{2 n-2} u^{2}}{2}\right\} \\
& \times \Phi\left\{\frac{n^{2}\left(1-y^{2}\right)^{5 / 2} y^{n-3} u}{2}\right\} d y \\
\leq & (2 \pi)^{-1} \exp \left\{-\frac{4 n^{2} \exp (-2 n / \log n) u^{2}}{(\log n)^{3}}\right\} \Phi\left\{\frac{2 \sqrt{2} n^{2} \exp \left(-n^{1-\alpha}\right) u}{n^{5 \alpha / 2}}\right\} \\
& \times \int_{1-(\log n)^{-1}}^{1-n^{-\alpha}} y^{-2}\left(1-y^{2}\right)^{-1} d y .
\end{aligned}
$$

We can tidy (3.35) to yield

$$
\begin{gathered}
(4 \pi)^{-1} \Phi\left\{\frac{2 \sqrt{2} n^{2} \exp (-n / \log n) u}{(\log n)^{5 / 2}}\right\} \exp \left\{-\frac{4\left(\log n^{k}\right)^{2} u^{2}}{n^{\alpha+2 k}}+\log \log n\right\} \\
\leq(2 \pi)^{-1} \int_{1-(\log n)^{-1}}^{1-n^{-\alpha}} y^{-2}\left(1-y^{2}\right)^{-1} \exp \left\{-\frac{n^{2}\left(1-y^{2}\right)^{3} y^{2 n-2} u^{2}}{2}\right\} \\
\times \Phi\left\{\frac{n^{2}\left(1-y^{2}\right)^{5 / 2} y^{n-3} u}{2}\right\} d y \\
\leq(4 \pi)^{-1} \Phi\left\{\frac{2 \sqrt{2}\left(\log n^{k}\right)^{2} u}{n^{(\alpha / 2)+k}}\right\} \exp \left\{-\frac{4 n^{2} \exp (-2 n / \log n) u^{2}}{(\log n)^{3}}+\log \log n\right\} .
\end{gathered}
$$

Clearly

$$
\begin{aligned}
& \int_{1+\varepsilon}^{\infty} I_{2}(x) d x \sim \frac{\log n}{8 \pi}, \quad \text { where } u=o\left(n^{k}\right), \\
& \int_{1+\varepsilon}^{\infty} I_{2}(x) d x \rightarrow 0, \quad \text { where } u \geq \frac{\exp (n / \log n)(\log n)^{5 / 2} f}{n^{2}},
\end{aligned}
$$

if $f$ is any function that tends to infinity as $n \rightarrow \infty$. Collecting together (3.31), (3.32), (3.37), and (3.38) we have proved a marginally more general result than is stated in Theorem 1.3. By increasing the level $u$ given in (3.32) and (3.38) a little to $u \geq$ $\exp (n / \log n)$, we obtain the result in Theorem 1.3 which can be more readily understood.

\section{REFERENCES}

[1] A. T. Bharucha-Reid and M. Sambandham, Random Polynomials, Probability and Mathematical Statistics, Academic Press, Inc., Orlando, Fla., 1986. MR 87m:60118. Zbl 615.60058 .

[2] H. Cramér and M. R. Leadbetter, Stationary and Related Stochastic Processes. Sample Function Properties and their Applications, John Wiley \& Sons, Inc., New York, London, Sydney, 1967. MR 36\#949. Zbl 162.21102. 
[3] M. Das, The average number of maxima of a random algebraic curve, Proc. Cambridge Philos. Soc. 65 (1969), 741-753. MR 39\#1026. Zbl 218.60017.

[4] K. Farahmand, On the average number of real roots of a random algebraic equation, Ann. Probab. 14 (1986), no. 2, 702-709. MR 87k:60140. Zbl 609.60074.

[5] _ Local maxima of a random trigonometric polynomial, J. Theoret. Probab. 7 (1994), no. 1, 175-185. MR 95b:60059. Zbl 786.60069.

[6] K. Farahmand and P. Hannigan, The expected number of local maxima of a random algebraic polynomial, J. Theoret. Probab. 10 (1997), no. 4, 991-1002. MR 99a:60053. Zbl 897.60055.

[7] K. Itô, The expected number of zeros of continuous stationary Gaussian processes, J. Math. Kyoto Univ. 3 (1963/1964), 207-216. MR 29\#4097. Zbl 139.34102.

[8] M. Kac, On the average number of real roots of a random algebraic equation, Bull. Amer. Math. Soc. 49 (1943), 314-320. MR 4,196d. Zbl 060.28602.

[9] M. R. Leadbetter, On crossings of levels and curves by a wide class of stochastic processes, Ann. Math. Statist. 37 (1966), 260-267. MR 34\#8476. Zbl 141.14906.

[10] J. E. Littlewood and A. C. Offord, On the number of real roots of a random algebraic equation, J. London Math. Soc. 13 (1938), 288-295. Zbl 020.13604.

[11] _ On the number of real roots of a random algebraic equation. II, Proc. Camb. Philos. Soc. 35 (1939), 133-148. Zbl 021.03702.

[12] A. C. Offord, The distribution of the values of an entire function whose coefficients are independent random variables. II, Math. Proc. Cambridge Philos. Soc. 118 (1995), no. 3, 527-542. MR 96m:30046. Zbl 846.60037.

[13] S. O. Rice, Mathematical analysis of random noise, Bell System Tech. J. 24 (1945), 46-156. MR 6,233i. Zbl 063.06487.

[14] J. E. Wilkins, Jr., An asymptotic expansion for the expected number of real zeros of a random polynomial, Proc. Amer. Math. Soc. 103 (1988), no. 4, 1249-1258. MR 90f:60105. Zbl 656.60062.

[15] N. D. Ylvisarer, The expected number of zeros of a stationary Gaussian process, Ann. Math. Statist. 36 (1965), 1043-1046. MR 31\#1721. Zbl 139.34201.

K. FARAHMAND: DEPARTMENT OF MATHEMATICS, UNIVERSITY OF UlSTER, JORDANSTOWN, CO. ANTRIM BT37 0QB, UK

E-mail address: k. farahmand@u1st.ac.uk

P. HANNigan: Department of MATHematics, University of Ulster, JoRdanstown, Co. ANTRIM BT37 0QB, UK 


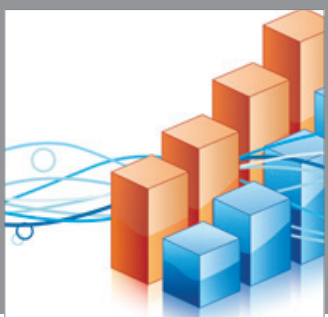

Advances in

Operations Research

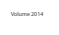

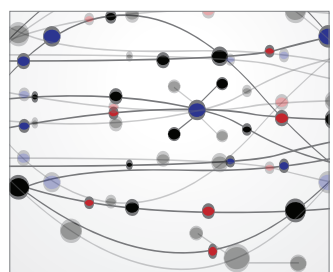

\section{The Scientific} World Journal
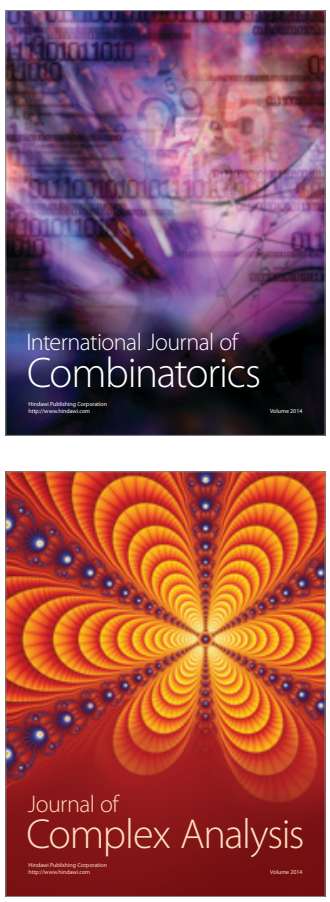

International Journal of

Mathematics and

Mathematical

Sciences
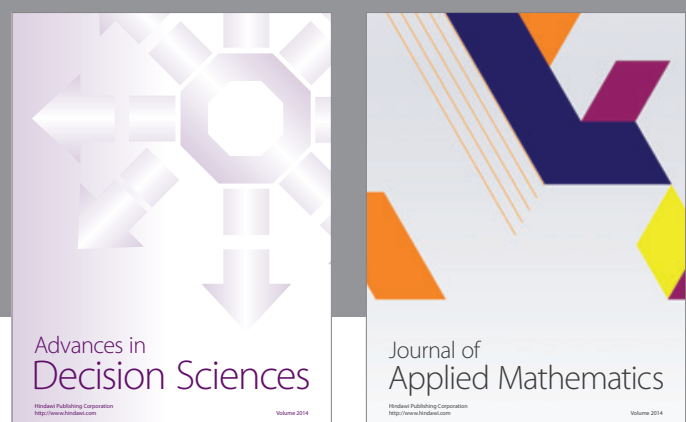

Journal of

Applied Mathematics
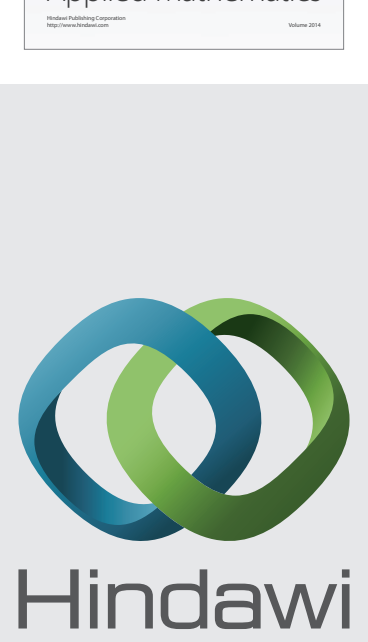

Submit your manuscripts at http://www.hindawi.com
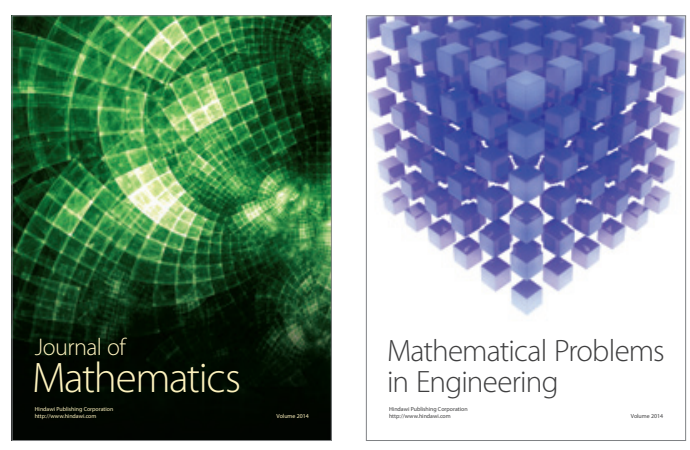

Mathematical Problems in Engineering
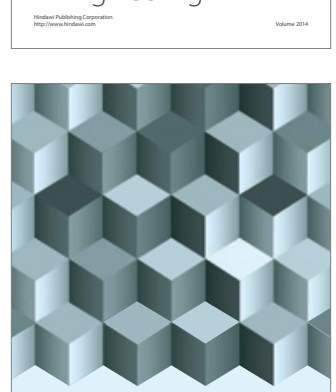

Journal of

Function Spaces
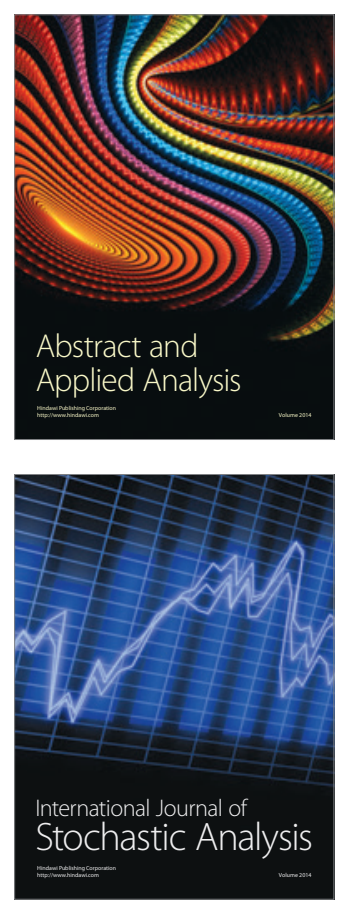

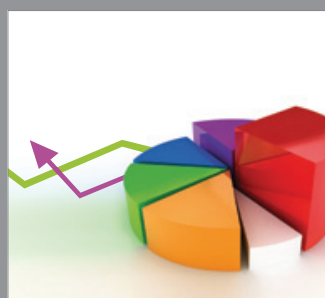

ournal of

Probability and Statistics

Promensencen
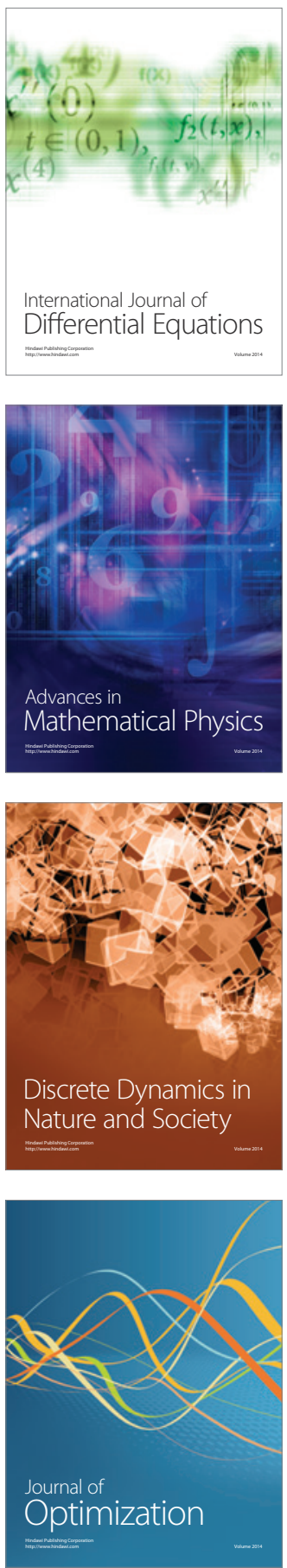